Result. There were 209 responses. This represents a response rate of approximately $10 \% .89 \%$ of respondents had completed a CRAFT tool at some point but only $38 \%$ had received training. $15 \%$ reported that the CRAFT did not aid decision making about risk in clinical settings, whereas $37 \%$ said it did and $42 \%$ said it did sometimes. $46 \%$ report patients are consulted most of the time $(34 \%)$ or always (12\%). The qualitative impression was that the CRAFT was an improvement on its predecessor. However common themes from responders highlighted a lack of clinical relevance or impact decision making, lack of training in filling it out and cumbersome integration with the electronic case notes.

Conclusion. Staff perceptions of the CRAFT tool were generally negative with many feeling it was a box ticking exercise that had minimal real world impact on patient risk and its management. However many felt it was an improvement over the previous risk tool and the majority used it at some point to aid clinical decision making.

\section{Medical comorbidities of patients presenting to an adult neurodevelopmental new case clinic in Singapore}

Ho Teck Tan ${ }^{1 \star}$, James Patrick Moon ${ }^{2}$ and Giles Ming Yee $\operatorname{Tan}^{1}$ ${ }^{1}$ Institute of Mental Health, Singapore and ${ }^{2}$ Sengkang General Hospital

${ }^{\star}$ Corresponding author.

doi: 10.1192/bjo.2021.782

Aims. To describe the occurrence of medical comorbity in patients with neurodevelopmental disorders presenting to the Adult Neurodevelopmental Service (ANDS) multi-disciplinary new case clinic at the Institute of Mental Health (IMH) in Singapore. We hypothesize that patients with neurodevelopmental disorders have higher rates of medical comorbidity compared to those without a diagnosis of neurodevelopmental disorder.

Background. Medical comorbidities are common in patients with neurodevelopmental disorders. They may have difficulties managing their medical conditions which could in turn affect their well being, quality of life and life expectancy.

Method. A retrospective cohort study was conducted amongst patients who presented to the clinic from January 2015 to December 2016. The electronic case records of the assessments were de-identified and the medical conditions of patients were collected and analysed.

Result. 319 patients attended the ANDS new case clinic in the 2-year study period. $87.1 \%(278 / 319)$ were diagnosed with a neurodevelopmental disorder while $12.9 \%$ (41/319) did not receive any diagnosis of a neurodevelopmental disorder.

$58.3 \%(162 / 278)$ of patients with a neurodevelopmental disorder had at least 1 medical comorbidity while only $31.7 \%$ (13/ 41) of patients with no neurodevelopmental disorder had at least 1 medical condition.

Patients with neurodevelopmental disorders had higher rates of epilepsy ( $12.2 \%$ vs $4.9 \%)$, cerebral palsy ( $3.2 \%$ vs $0 \%$ ) but lower rates of having other neurological conditions $(1.4 \%$ vs $7.3 \%)$ compared to those with no neurodevelopmental disorders.

Patients with neurodevelopmental disorders had higher rates of diabetes $(6.1 \%$ vs $2.4 \%)$, hypertension (6.1\% vs $2.4 \%)$, hyperlipidaemia ( $7.1 \%$ vs $2.4 \%)$ and cardiovascular conditions $(2.9 \%$ vs $0 \%$ ) than those without a neurodevelopmental disorder.

In terms of other medical comorbidities, patients with neurodevelopmental disorders had higher rates of thyroid abnormalities $(4.7 \%$ vs $2.4 \%)$, respiratory problems ( $7.6 \%$ vs $2.4 \%)$, musculoskeletal conditions ( $5.8 \%$ vs $0 \%$ ), eye issues ( $5 \%$ vs $2.4 \%$ ) and hearing problems (2.9\% vs $0 \%$ ) but similar rates of dermatological conditions (10.1\% vs $9.8 \%)$ and gastrointestinal conditions $(4.7 \%$ vs $4.9 \%)$ compared to those with no neurodevelopmental disorders.

Conclusion. Patients with neurodevelopmental disorders have significantly highly rates of medical comorbidity than those without any neurodevelopmental disorders. This study highlights the need to raise awareness of the common medical comorbidities in patients with neurodevelopmental disorders and to ensure adequate screening and referral for follow-up medical care for them.

\section{Interview skills - psychiatry reel to reality}

Mathuri Tharmapoopathy ${ }^{1 \star}$, Santosh Kumar ${ }^{2}$ and Abishan Thavarajah ${ }^{3}$

${ }^{1}$ Newcastle University Medical school; ${ }^{2}$ Roseberry Park Hopsital, Tees, Esk and Wear Valley NHS trust and ${ }^{3}$ Queen Mary University and Barts and the London School of Medicine and Dentistry ${ }^{\star}$ Corresponding author.

doi: 10.1192/bjo.2021.783

Aims. This reel analysis identifies quotes and actions of fictional characters from TV shows, namely: Hercules Poirot, Sherlock Holmes and House who can demonstrate learning points for clinical students to use within real psychiatric practice, using scientific theories such as the Hypothetico-deductive model, Empirical falsification and Occam's razor. This analysis explores what an ideal psychiatric interview consists of and what can be learnt from these characters and implemented within medical education.

Method. Each show was watched by one researcher over the period of March to August 2020. The researcher noted insightful quotes which were relevant to one of the three philosophical theories. Quotes were included if they demonstrated deduction skills, revealed a character's ethos and supported the CalgaryCambridge model of interviewing such as building rapport. 32 quotations were collected in total and narrowed to 6 quotations. These were then analysed, learning points were made and linked to the Calgary Cambridge model.

Result. Dr House demonstrates objectivity when taking a patient's history. He utilises empirical falsification when diagnosing to avoid missing a differential diagnosis. Detective Poirot displays how empathic listening allows disclosure of details in the history, which would have otherwise been omitted. Additionally, he illustrates the importance of collateral interviewing which allows one to identify misinterpretations and inconsistencies. Sherlock teaches us the importance of perception regarding mismatching information which can help to gather new facts. All three characters interview beginning with open questions to more closed questions, supplementing with deductive reasoning in order to solve cases. Objectivity, empirical falsification, empathetic listening and deductive reasoning are the key skills displayed by these characters, that medical students can most use in their own practices.

Conclusion. The perfect interview discovers new information through synchronised collaboration, whilst adhering to the Hypothetico-deductive model of thought. A combination of the Calgary-Cambridge model of interviewing and skillset of the TV characters should be considered for implementation in some aspects of psychiatric interviewing. Medical education can utilise these TV shows to teach students how to conduct history-taking. 